\title{
Lesões orais secundárias à leishmaniose visceral em um cão com hiperadrenocorticismo endógeno: relato de caso
}

[Oral lesions secondary to visceral leishmaniasis in a dog with endogenous hyperadrenocorticism: case report]

\author{
M.S. Bochnakian ${ }^{1}$, J.A.B. Bezerra ${ }^{1 *}$, K.S.B.R. Fernandes ${ }^{1}$, V.R. Barros ${ }^{1}$, V.V. Paula ${ }^{1}$, \\ J.P.S. Santos ${ }^{2}$, K.D. Filgueira ${ }^{1}$ \\ ${ }^{1}$ Universidade Federal Rural do Semiárido - Mossoró, RN \\ ${ }^{2}$ Médica Veterinária Autônoma - Clínica Veterinária Nobreza Canina - Mossoró, RN
}

\begin{abstract}
RESUMO
O objetivo do presente trabalho foi relatar um caso de leishmaniose visceral com apresentação mucosa em um cão com hiperadrenocorticismo. Um canino, macho, da raça Poodle, 11 anos de idade, foi atendido com histórico de disfagia, halitose e sialorreia. Ao exame físico, observou-se linfadenomegalia generalizada e alterações cutâneas, como rarefação pilosa, comedões, telangiectasia e atrofia cutânea. Além disso, o animal também apresentava formações orais localizadas na língua. Dos exames hematológicos e bioquímicos realizados, a única alteração encontrada foi elevação da fosfatase alcalina $(1724 u / L)$. O teste de supressão com a dexametasona em dose baixa foi executado para investigar hiperadrenocorticismo, tendo resultado positivo. Também foram realizados exames citológicos dos linfonodos, da medula óssea e das formações orais, tendo sido observada a presença de formas amastigotas de Leishmania sp. em todas as amostras. $\mathrm{O}$ animal foi submetido à biópsia incisional das formações orais, e a análise histopatológica demonstrou um quadro de inflamação granulomatosa com presença de grande quantidade de microrganismos morfologicamente compatíveis com formas amastigotas de Leishmania sp. no interior das células inflamatórias. Diante dos achados clínicos e dos exames complementares, diagnosticou-se um caso de leishmaniose com manifestação mucosa atípica, associado ao hiperadrenocorticismo, podendo essa endocrinopatia ter sido um fator predisponente para essa enfermidade infectocontagiosa.
\end{abstract}

Palavras-chave: Leishmania infantum, leishmaniose oral, imunossupressão

\section{ABSTRACT}

The objective of the present study was to report a case of visceral leishmaniasis with mucosal presentation in a dog with hyperadrenocorticism. A canine, male, Poodle, 11 years old, was attended with a history of dysphagia, halitosis, and sialorreia. The physical examination revealed generalized lymphadenomegaly and cutaneous alterations such as hair loss, comedones, telangiectasia, and cutaneous atrophy. Futhermore, the animal also had localized oral formations on the tongue. From the hematological and biochemical tests performed, the only alteration was alkaline phosphatase elevation $(1724 u$ / L). The low dose dexamethasone suppression test was performed to investigate hyperadrenocorticism and found a positive result. In addition, cytological exams of lymph nodes, bone marrow and oral formations were also performed, and the presence of amastigote forms of Leishmania sp. were observed in all samples. The animal was submitted to incisional biopsy of the oral formations and the histopathological analysis showed a granulomatous inflammation with presence of large quantity of microorganisms morphologically compatible with amastigotes forms of Leishmania sp. within the inflammatory cells. Faced with clinical findings and complementary exams, a case of leishmaniasis with atypical mucosal manifestation, associated with hyperadrenocorticism, was diagnosed, and this endocrinopathy could have been a predisposing factor to this infectious-contagious disease.

Keywords: Leishmania infantum, oral leishmaniasis, immunosuppression

Recebido em 12 de agosto de 2017

Aceito em 17 de novembro de 2017

* Autor para correspondência (corresponding author)

E-mail: artur_brilhante@hotmail.com 


\section{INTRODUÇÃO}

A leishmaniose visceral canina (LVC) é uma doença infecciosa crônica, causada pelo protozoário Leishmania infantum, que pode desencadear uma grande variedade de sinais clínicos (Solano-Gallego et al., 2011). De forma atípica, alguns cães infectados por esse parasita desenvolvem a forma mucosa da doença, levando ao surgimento de lesões orais, localizadas principalmente na língua (Foglia Manzillo et al., 2009). Essas lesões possuem apresentações macroscópicas distintas, sendo desde pápulas e placas até nódulos ou tumores não neoplásicos, que podem aparecer como único sinal clínico da LVC, dificultando a suspeição inicial para tal enfermidade (Lamouthe e Poujade, 2002; Foglia Manzillo et al., 2005; Parpaglia et al., 2007; Viegas et al., 2012; Tangalidi et al., 2016).

Em regiões endêmicas para a LVC, devido à elevada prevalência e ao caráter crônico da doença, a relação com outras enfermidades é uma realidade em potencial (Saridomichelakis, 2009). Dentre essas, merecem destaque as doenças que culminam com imunossupressão, a exemplo de neoplasias e certas endocrinopatias, como o hiperadrenocorticismo (HAC) (SolanoGallego et al., 2011).

No HAC de ocorrência natural, observa-se um aumento persistente nas concentrações sanguíneas de cortisol, ocasionando uma série de alterações sistêmicas, incluindo disfunções imunológicas que predispõem a infecções fúngicas, bacterianas, virais e parasitárias (Pereira et al., 2007; de Marco, 2015). Dessa forma, objetivou-se relatar um caso de leishmaniose com apresentação mucosa em um cão com HAC.

\section{RELATO DE CASO}

Um canino, macho, da raça Poodle, 11 anos de idade, autóctone da cidade de Mossoró (estado do Rio Grande do Norte, Brasil), foi atendido com histórico de disfagia, halitose e sialorreia. Não havia relato de terapias pregressas. Submeteu-se o paciente ao exame físico. Os parâmetros fisiológicos encontravam-se dentro da normalidade. Entretanto, na inspeção cutânea, o animal apresentava rarefação pilosa em região sacral, além de comedões, telangiectasia e atrofia cutânea (pele “pregueável” ou “papiriforme”) em região abdominal ventral e inguinal. Ocorria ainda linfadenomegalia generalizada.

Na avaliação da cavidade oral, observou-se doença periodontal. No antímero direito, havia uma placa localizada entre a face ventral da língua e o assoalho bucal, com envolvimento do frênulo lingual. Essa formação apresentava dimensões de 6,3 x 1,3 x 0,9cm e estendia-se desde os dentes pré-molares até os molares inferiores direitos, mas sem envolvimento visível deles (Fig. 1). Além dessa lesão, existiam ainda nódulos, no terço médio da face lateral direita da língua, sendo o maior com as dimensões de 1,3 $\mathrm{x}$ $0,9 \times 0,76 \mathrm{~cm}$ (Fig. 1). Ambas as lesões demonstravam consistência firme, base de inserção séssil, forma irregular e superfície externa erodida. Não foram observadas alterações significativas nos demais componentes da cavidade oral ou em outras estruturas orgânicas aparentes.

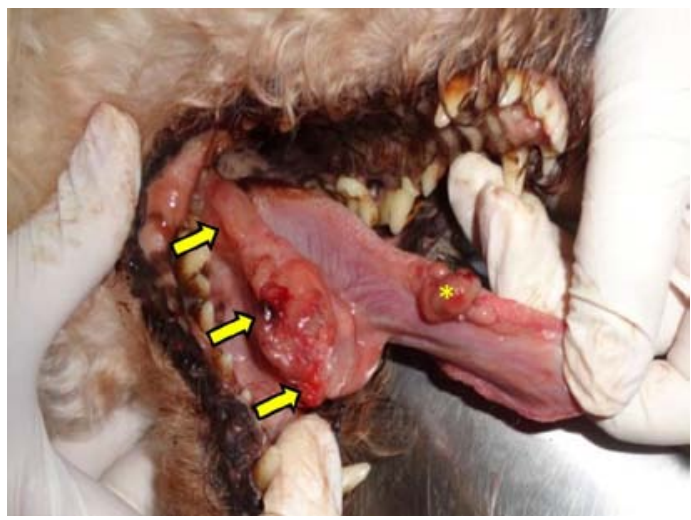

Figura 1. Apresentação macroscópica das formações orais de canino macho, raça Poodle, 11 anos de idade. Verifica-se uma placa entre a face ventral da língua e o assoalho bucal (setas) e nódulos na face lateral direita do referido órgão (asterisco).

Como exames complementares, foram solicitados hemograma completo e perfil bioquímico sérico, composto pela análise da alanina aminotransferase, aspartato aminotransferase, fosfatase alcalina, ureia, creatinina, proteínas totais e frações, além de colesterol, triglicerídeos e glicose. Investigou-se ainda a possível elevação do cortisol sistêmico, com o auxílio do teste de supressão com a dexametasona em dose baixa. Optou-se por realizar exame citológico dos linfonodos, medula 
óssea e formações orais. Para essas últimas, também se executou biopsia incisional, cujas amostras foram destinadas à histopatologia.

A avaliação hematológica não exibiu alterações significativas. $\mathrm{Na}$ análise bioquímica, a única anormalidade equivaleu ao aumento dos níveis da fosfatase alcalina $(1724 \mathrm{u} / \mathrm{L})$. No teste de supressão com a dexametasona em dose baixa, observou-se um valor de cortisol basal 41,5ng/mL, e, após 8 horas da administração da dexametasona, o cortisol passou para 49,5ng/mL, sendo, neste caso, positivo para HAC endógeno. $\mathrm{Na}$ citopatologia dos linfonodos, da medula óssea e das lesões bucais, foi observada a presença de formas amastigotas de Leishmania sp. (Fig. 2). No exame histopatológico oral, o

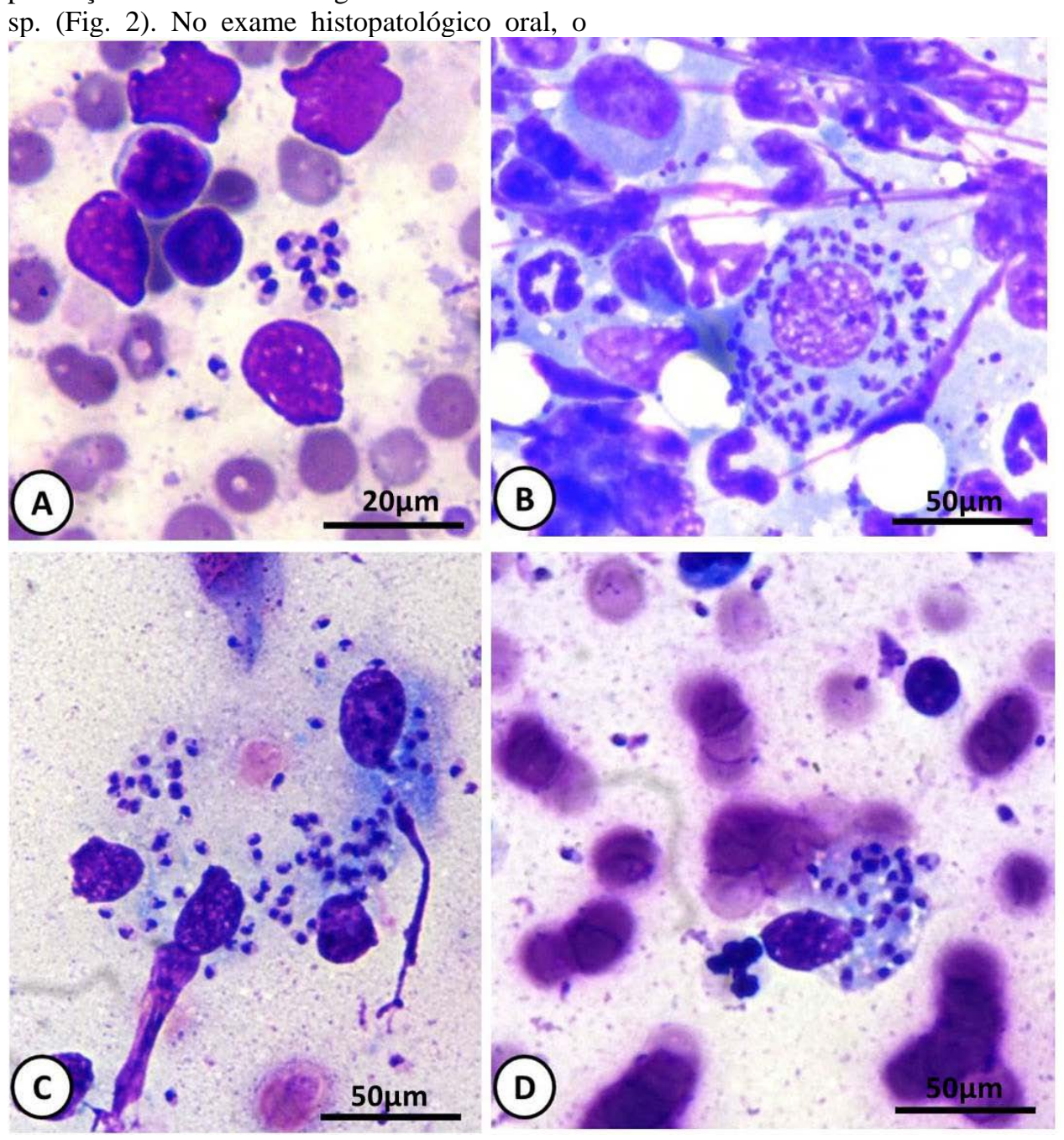

Figura 2. Fotomicrografias citopatológicas evidenciando a presença de formas amastigotas de Leishmania sp. em distintos locais em canino macho, raça Poodle, 11 anos de idade. A: linfonodo (Objetiva 100x. Coloração rápida: Romanowsky) B: medula óssea (Objetiva 40x. Coloração rápida: Romanowsky). C e D: placa e nódulo lingual, respectivamente (Objetiva 40x. Coloração rápida: Romanowsky). 


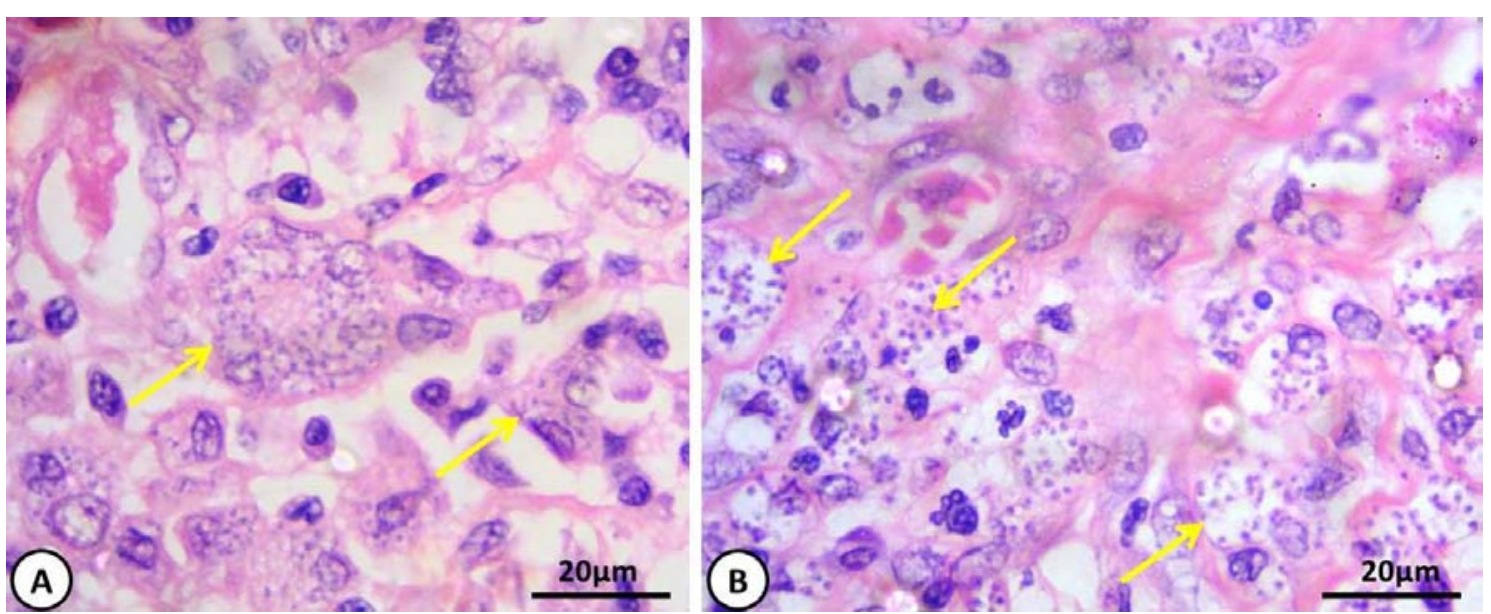

Figura 3. Fotomicrografias histopatológicas de formações orais de canino macho, raça Poode, 11 anos de idade. Demonstra-se a ocorrência de tecido conjuntivo, infiltrado mononuclear e polimorfonuclear, em associação a formas amastigotas de Leishmania sp. (setas) no interior do citoplasma de macrófagos e células epitelioides. A e B: placa e nódulo lingual, respectivamente (Objetiva 100x. Coloração: hematoxilina e eosina).

\section{DISCUSSÃO}

A presença de lesões orais na LVC é considerada rara, e poucos trabalhos descrevem tal apresentação na literatura (Lamouthe e Poujade, 2002; Foglia Manzillo et al., 2005; Parpaglia et al., 2007; Foglia Manzillo et al., 2009; Viegas et al., 2012; Tangalidi et al., 2016). Nos escassos relatos dessa forma clínica atípica da LVC, as lesões foram caracterizadas por únicos ou múltiplos nódulos em toda a superfície da língua, além de úlceras e pápulas, corroborando os achados do presente caso (Lamouthe e Poujade, 2002; Foglia Manzillo et al., 2005; Parpaglia et al., 2007; Foglia Manzillo et al., 2009; Viegas et al., 2012; Tangalidi et al., 2016). De modo geral, nos casos de LVC que envolvem lesões na cavidade oral, também há manifestação de sinais em outros órgãos ou sistemas (Parpaglia et al., 2007; Foglia Manzillo et al., 2009; Viegas et al., 2012). Entretanto, já foi descrito um caso em que o único sinal clínico apresentado pelo animal acometido por LVC equivaleu a lesões linguais (Tangalidi et al., 2016), assim como observado no cão em discussão. O cão relatado não exibia sinais clássicos para a LVC, o que tornou os exames citológico e histopatológico das lesões orais essenciais para o direcionamento do diagnóstico e a confirmação da presença do protozoário.

Tangadili e colaboradores (2016) justificaram o acometimento oral de um cão com leishmaniose mediante a hipótese da ingestão acidental de flebótomos infectados, que levou à inoculação do protozoário e desencadeou uma reação inflamatória local devido à multiplicação da Leishmania, resultando no surgimento da lesão. Quando há formações nodulares no sítio de infiltração dos parasitos do gênero Leishmania, a lesão é denominada de "leishmanioma” ou “cancro de inoculação” (Blavier et al., 2001). Essa hipótese pode ser levada em consideração para explicar o caso aqui descrito, em que estava presente uma doença periodontal considerável, que pode ter atraído os flebótomos para a boca do animal devido ao acúmulo de material orgânico na cavidade oral. Outra possibilidade seria que as lesões na língua teriam ocorrido de forma secundária, após a disseminação sistêmica do parasito, já que foi comprovado o envolvimento da medula óssea e de linfonodos, por meio do exame citológico.

Os diagnósticos diferenciais a serem considerados em casos de LVC com manifestações na cavidade oral são granuloma eosinofílico, amiloidose mucocutânea, micoses sistêmicas, linfoma e outros processos inflamatórios e neoplásicos (Blavier et al., 2001; Tangadili et al., 2016). Nesse sentido, a histopatologia foi essencial no relato em questão, uma vez que possibilitou a exclusão por definitivo de outros processos proliferativos que poderiam ser correlacionados com o leishmanioma oral. 
A associação entre a LVC e as doenças endócrinas ainda é pouco elucidada (Cortese et al., 1999). Ao conhecimento dos autores, há apenas um trabalho descrevendo a ocorrência de tal enfermidade infecciosa juntamente com o HAC, em que foram constatadas formas amastigotas do gênero Leishmania em exame histopatológico de um adenoma adrenocortical (Ferro et al., 2013). No HAC, em razão dos elevados níveis de cortisol circulante, há um quadro de imunossupressão devido ao comprometimento das respostas celulares da reação inflamatória e à diminuição do número e da função dos linfócitos (Pereira et al., 2007). Assim, em condições de imunodepressão, como no paciente em discussão, os riscos de se adquirir a LVC, especialmente em áreas endêmicas, são elevados, e formas não usuais da doença podem se desenvolver (Pittalis et al., 2006). Um caso semelhante ao do presente relato foi descrito em um humano, o qual recebia corticoterapia crônica, e acabou adquirindo a leishmaniose, com manifestação de lesões ulcerativas na língua (Pittalis et al., 2006).

\section{CONCLUSÃO}

Em cães que apresentem lesões na cavidade oral, em especial na língua, e que sejam oriundos de áreas endêmicas para a leishmaniose visceral, é de fundamental importância considerar essa parasitose como potencial diagnóstico diferencial. Além disso, deve-se considerar o HAC como fator de risco para o contágio da LVC, devido às implicações deletérias que tal endocrinopatia exerce sobre o sistema imunológico.

\section{REFERÊNCIAS}

BLAVIER, A.; KEROACK, S.; DENEROLLE, P. et al. Atypical forms of canine leishmaniosis. Vet. J., v.162, p.108-20, 2001.

CORTESE, L.; OLIVA, G.; CIARAMELLA, P. et al. Primary hypothyroidism associated with leishmaniasis in a dog. J. Am. Anim. Hosp. Assoc., v.35, p.487-492, 1999.

DE MARCO, V. Dermatopatias Hormonais: Hiperadrenocorticismo. In: LARSSON, C.E.; LUCAS, R. Tratado de medicina externa: dermatologia veterinária. São Paulo: Interbook, 2015, p.575-591.
FERRO, S.; PALMIERI, C.; CAVICCHIOLI, L. et al. Leishmania amastigotes in neoplastic cells of 3 nonhistiocytic canine tumors. Vet. Pathol., v.50, p.749-752, 2013.

FOGLIA MANZILLO V.; PAPARCONE, R.; CAPPIELLO, S. et al. Resolution of tongue lesions caused by Leishmania infantum in a dog treated with the association miltefosineallopurinol. Parasitol. Vectors, v.2, Supl.1, p.S6, 2009.

FOGLIA MANZILLO, V.; PAGANO, A.; PACIELLO, O. et al. Papular-like glossitis in a dog with leishmaniosis. Vet. Rec., v.156, p.213215, 2005.

LAMOTHE, J.; POUJADE, A. Ulcerative glossitis in a dog with leishmaniasis. Vet. Rec., v.151, p.182-183, 2002.

PARPAGLIA, M.L.; VERCELLI, A.; COCCO, R. et al. Nodular lesions of the tongue in canine leishmaniosis. J. Vet. Med. A. Physiol. Pathol. Clin. Med., v.54, p.414-417, 2007.

PEREIRA, A.L.C.; BOLZANI, F.C.B.; STEFANI, M. et al. Uso sistêmico de corticosteróides: revisão da literatura. Med. Cutan. Ibero Lat. Am., v.35, p.35-50, 2007.

PITTALIS, S.; NICASTRI, E.; SPINAZZOLA, F. et al. Leishmania infantum leishmaniasis in corticosteroid-treated patients. BMC Infect. Dis., v.18, p.177, 2006.

SARIDOMICHELAKIS, M. Advances in the pathogenesis of canine leishmaniosis: epidemiologic and diagnostic implications. Vet. Dermatol., v.20, p.471-489, 2009.

SOLANO-GALLEGO, L.; MIRÓ, G.; KOUTINAS, A. et al. LeishVet guidelines for the practical management of canine leishmaniosis. Parasitol. Vectors, v.86, p.1-16, 2011.

TANGALIDI, M.K.; OIKONOMIDIS, I.L.; PSALLA, D. et al. Nodular granulomatous glossitis as the sole clinical sign in canine leishmaniosis. Vet. Clin. Pathol., v.45, p.710714, 2016.

VIEGAS, C.; REQUICHA, J.; ALBUQUERQUE, C. et al. Tongue nodules in canine leishmaniosis - a case report. Parasitol. Vectors, v.120, p.1-5, 2012. 